\title{
Effects of a Single Physical or Mindfulness Intervention on Mood, Attention, and Executive Functions: Results from two Randomized Controlled Studies in University Classes
}

\author{
Christian Müller ${ }^{1,2}$ (D) David Dubiel ${ }^{3} \cdot$ Eleni Kremeti $^{4} \cdot$ Manuel Lieb $^{5} \cdot$ Elisabeth Streicher $^{5} \cdot$ Nour Siakir Oglou $^{6}$. \\ ${\text { Christoph } \text { Mickel }^{3} \cdot \text { Julia Karbach }}^{2,7}$
}

Accepted: 15 February 2021 / Published online: 15 March 2021

(C) The Author(s) 2021

\begin{abstract}
Objectives The primary objective of this study was to examine the effects of one-session physical or mindfulness training on university students' mood, attention and executive functions in two separate randomized studies.

Methods Study 1 (physical activity intervention) was implemented in a seminar with 63 and Study 2 (mindfulness intervention) in another seminar with 28 university students. The physical intervention included stretching exercises, balancing tasks, and medium intensity cardiovascular activities. The mindfulness training included yoga exercises, guided attention, and a body scan. In the control conditions, students watched a 15-min fitness or yoga video, respectively. Several mood and attention scales, as well as executive functions were assessed before and after the intervention or control activity. A randomized within-subject crossover design was applied in both studies.

Results Repeated-measures analysis of variance revealed that participants in both intervention conditions reported mood to be more positive, more awake and calmer after the intervention compared to the control conditions. These effects were medium to large (Study 1: eta $^{2}=.08-.30$, Study 2: eta $^{2}=.15-.30$ ). Attention scores improved more relative to the control condition after the physical intervention (medium effect size, eta ${ }^{2}=.11$ ). Executive function scores improved more relative to the control condition after the mindfulness intervention (medium effect size, eta ${ }^{2}=.17$ ).

Conclusions These results indicate that a short bout (15-min) of physical or mindfulness activity in a university learning setting positively affected dimensions of mood and cognition known to support academic learning.
\end{abstract}

Keywords Physical exercise $\cdot$ Mindfulness intervention $\cdot$ University learning $\cdot$ Classroom-based intervention $\cdot$ Mood $\cdot$ Attention . Executive functions

Christian Müller

c.mueller@psychologie.tu-darmstadt.de

1 Institute of Psychology, Technische Universität Darmstadt, Alexanderstraße 10, 64283 Darmstadt, Germany

2 Center for Individual Development and Adaptive Education of Children at Risk (IDeA), Frankfurt am Main, Germany

3 Institute of Sports Sciences, Goethe-University, Frankfurt am Main, Germany

4 Department of Psychology, Philipps-University Marburg, Marburg, Germany

5 Institute of Psychology, Goethe-University, Frankfurt am Main, Germany

6 Department of Psychology, Ege University, Bornova-Izmir, Turkey

7 Department of Psychology, University of Koblenz-Landau, Landau / Pfalz, Germany
Previous research has identified several cognitive and affective variables associated with successful learning, such as executive functions, attention, and mood (Titz and Karbach 2014; Tyng et al. 2017). However, these individual resources are limited and influenced by internal and external conditions (Allport 1980; Kahneman 1973; Nadler et al. 2010; Reeve 2014). On the cognitive side, the ability to flexibly switch attention to relevant information and to remain focused on a task (Driver 2001; Lavie 2005) in the presence of distractors is constantly challenged in institutional learning settings (Farley et al. 2013; Lindquist and McLean 2011). On the affective side, research showed that positive mood is associated with beneficial learning strategies and facilitates the integration of new information into existing memory content (Ainley 2006; Ashby et al. 1999). Yet inter-individual changes in mood are considerable and students often report being bored in 
institutional learning settings (Pekrun et al. 2010; Sharp et al. 2017; Wilson and Korn 2007). One way to improve these learning conditions are breaks (Ramstetter et al. 2010), such as physical activity and mindfulness interventions.

There is growing evidence for positive effects of brief (one session) bouts of physical exercise on cognitive performance (Bailey et al. 2014; Brisswalter et al. 2002; Chang et al. 2012; Lambourne and Tomporowski 2010; Roig et al. 2013; Tomporowski 2003; Van den Berg et al. 2016; Zinke et al. 2012). Meta-analyses on the effects of acute exercise on cognitive performance, such as information processing, speeded performance, attention, and executive functions, also indicated overall positive but small effects (Chang et al. 2012; Lambourne and Tomporowski 2010). Looking specifically at attention and executive functions, a recent review on the effects of short bouts of physical activity based on 12 studies with children and adolescents ( $7-17$ years) provided mixed results, with improvements on measures of attentional control in lab-based studies but inconclusive results in school-based studies (Janssen et al. 2014). Moreover, the findings suggested that the effects may vary as a function of the participant's age, exercise type, and duration. Performance benefits after short physical interventions have also been reported for the three core domains of executive functioning (working memory, inhibition, and flexibility; Miyake et al. 2000). For instance, a recent study showed that a single bout of moderate physical activity benefited inhibition skills directly after the intervention (compared to a control condition watching a sports video; Spitzer and Furtner 2016). For shifting, a number of studies reported positive effects after long- and short-term interventions of physical activity (Barenberg et al. 2011; Berse et al. 2015; S.-R. Chen et al. 2016). For updating, Pontifex et al. (2009) found improvements immediately after an acute bout of aerobic exercise. Studies investigating all three executive core domains simultaneously showed mixed results. Some report better performance on tasks assessing all three aspects of executive functions after $30 \mathrm{~min}$ of acute moderate exercise (A.-G. Chen et al. 2014), others report no such improvement (Jäger et al. 2015).

More and more mindfulness-based interventions have been applied in non-clinical samples in order to improve information processing, cognition, or attentional processes (Jha et al. 2007; Napoli et al. 2005; Verhaeghen 2016). Mindfulnessbased interventions usually require repeated mindfulness practice (Cahn and Polich 2006) and the majority of mindfulness studies included multiple sessions over the course of several weeks (Nyklíček and Kuijpers 2008). Mindfulnessbased stress reduction (MBSR) programs usually last 8 weeks and combine yoga, perception, and guided attention exercises (Kabat-Zinn 2003). Research investigating the effects of single mindfulness sessions on cognition (e.g., Taraban et al. 2017) is rare compared to single physical activity session studies on that topic. Additionally, existing single-session mindfulness studies differ vastly in quality and intervention durations (Goldberg et al. 2017; van Dam et al. 2018), or focus on clinical outcomes, such as stress reduction (Rausch et al. 2006) or emotion regulation (Erisman and Roemer 2010; Fennell et al. 2016). Existing mindfulness studies report improved attentional control skills, with experienced meditators performing better on attentional tasks than participants with no meditation experience (e.g., Jha et al. 2007; Moore and Malinowski 2009; Sauer et al. 2012; Slagter et al. 2007). A more recent review on evidence for mindfulness meditationrelated benefits on executive functioning included 12 studies (Gallant 2016). Results of the review suggested consistent inhibitory improvement, but less consistent findings with respect to updating and shifting. A study by Mrazek et al. (2012) focusing on attention demonstrated that a single 8-min mindfulness intervention with undergraduate students reduced mind-wandering and improved attention compared to a passive control and a reading control group. Another study with 32 male students (without meditation experience) revealed improved memory compared to an active control group after a 20-min mindfulness intervention (Mohan et al. 2011). Interestingly, there is also evidence for individual differences in training outcomes. When participants in a brief 10-min mindfulness intervention condition were compared to a podcast-listening group and to a group completing arithmetic exercises, the results revealed that participants with low mindfulness trait (i.e., novices) showed the largest inhibition improvements (Watier and Dubois 2016).

Very few studies aimed at improving cognitive outcome measures have directly compared physical and mindfulness interventions. Gothe et al. (2013) examined the immediate effects of a single yoga session and a single aerobic exercise session on executive functions and working memory in a repeated-measures within-subjects design. They found that executive functions improved after yoga exercises (as compared to an active control condition), but not after aerobic exercises.

In sum, there is evidence for positive effects of singlesession physical exercise or mindfulness interventions on attention and executive functions, but results are heterogeneous and previous studies differ significantly in terms of design and methodology. Consequently, the comparability of their results is limited and there is a need for studies directly comparing the effects of both types of interventions across the same set of outcome measures and participants. In addition, most of the existing studies have assessed cognitive performance in labbased settings; therefore, results for classroom-based interventions are still scarce.

Aside from effects on cognitive processes, previous studies have also examined the impact of physical exercise and mindfulness interventions on mood. This is of particular interest for classroom-based interventions because mood is also associated with cognition and learning outcomes (Isen 1999; Nadler 
et al. 2010). Individual studies (Hansen et al. 2001; Scully et al. 1998), as well as reviews and meta-analyses (Penedo and Dahn 2005; Reed and Ones 2006), indicated that physical exercise positively affected mood. One meta-analysis showed that single sessions with acute low-to-moderate exercises of up to $35 \mathrm{~min}$ as well as shorter 15 -min bouts of moderate physical exercise improved mood (Reed and Ones 2006). This is consistent with a study from Wichers et al. (2012) showing increased positive affect but no change in negative affect after increased physical activity in daily life.

Mindfulness meditation has also been shown to improve mood (Bishop et al. 2004; Luu and Hall 2017). Reviews concluded that mindfulness-based interventions (more than one session) have moderate beneficial effects on anxiety and mood symptoms (Hofmann et al. 2010), and emotional reactivity (Lin et al. 2011) as well as on preventing depressive moods (Chong et al. 2011; Cramer et al. 2013; Fjorback et al. 2011). Mindfulness studies have rarely investigated the effects of single-session mindfulness training in non-clinical settings. For example, Taraban et al. (2017) investigated mindfulness effects on attention and mood of university students in a laboratory setting and reported more positive affect, less mind wandering, and a raised mindfulness state after $12 \mathrm{~min}$ of attention training.

Overall, previous evidence indicated that there are positive effects of both physical exercise and mindfulness training on mood. However, existing studies differ vastly in terms of methodology, samples, intervention content, and the way mood has been assessed. Moreover, none of them have examined the effects of short physical and mindfulness interventions on mood in the classroom.

The present study aimed to test whether one brief session of physical exercise or mindfulness meditation affected cognition (attention, executive function) and mood in the classroom. By implementing both interventions in comparable settings (university classes) and applying the same set of measures at pretest and posttest, we increased the comparability of findings. In two controlled studies, we assessed the effects of a short bout of physical exercise (Study 1) and mindfulness intervention in university classes (Study 2) on attention, executive function, and mood as compared to control condition (watching a video). Based on previous findings, we expected positive effects of physical exercise (Ludyga et al. 2016) and mindfulness intervention (Watier \& Dubois, 2016) on cognitive abilities and mood.

\section{Method}

\section{Participants}

Study 1 (physical activity intervention) included 63 university students (mean age: 25.85 years, $S D=5.06,81 \%$ female) and
Study 2 (mindfulness intervention) included 28 university students (mean age 23.69 years, $S D=3.12$, 68\% female). Students were informed about the study by research assistants 4-6 weeks in advance during an organizational meeting of the course. All participants were students of a major German university participating in mandatory classes on educational science. Classes included students from different tracks of teacher-training (elementary school to high school) and with different study majors. Students participated in full-day classes ( $6-8 \mathrm{~h}$ per day) which were part of their university education. Study participation was voluntary and participants provided written informed consent. No one dropped out and none of the students participated in both studies.

Using $\mathrm{G}^{*}$ Power (Faul et al. 2007), an a-priori power analysis with the parameters alpha $=.05$, power $=0.90$, eta-square $=.05$, indicated that a sample size of $N=52$ was necessary to test the effects of interest. Thus, Study 1 was well-powered. For Study 2, a post hoc power analysis, with the parameters alpha level $=.05$, sample size $=28$, and effect sizes $=.15-.30$, indicated that the power was sufficient in this study as well $(0.85-0.99)$.

\section{Procedure}

A randomized within-subject cross-over design was applied in both studies (see Fig. 1). On day 1, participants performed a pretest, the intervention (group A) or the control activity (group B), and a posttest. On day 2, participants completed the same assessment with a reversed intervention assignment (i.e., participants in group A completed the control activity and participants in group B completed the intervention; see Fig. 1). This design is particularly useful for interventions with short-term effects and little carry-over effects, as expected in the present study. In order to be able to control for carry-over effects, we performed pretest assessments on each test day.

The two studies included two different samples, and were implemented in two separate university classes. Assessments took place on two consecutive days of full-day seminars and after 120 min of class on each day. At pretest on day 1, participants were not aware of the condition they would be assigned to later. The allocation to intervention or control condition was quasi-randomized (matched by gender). Interventions were conducted in separate rooms of the university similar in size and interior. On the second day, students completed the same assessment with a reversed group assignment (see above and Fig. 1) to complete the cross-over design. The same trained research assistants were in charge of the assessments on both days of the study. The sessions started with the pretest $(15 \mathrm{~min})$, including the mood questionnaire followed by the attention test and executive function task. After the pretest, participants performed the 15-min intervention (physical or mindfulness training) or the control activity 
Day 1

Day 2

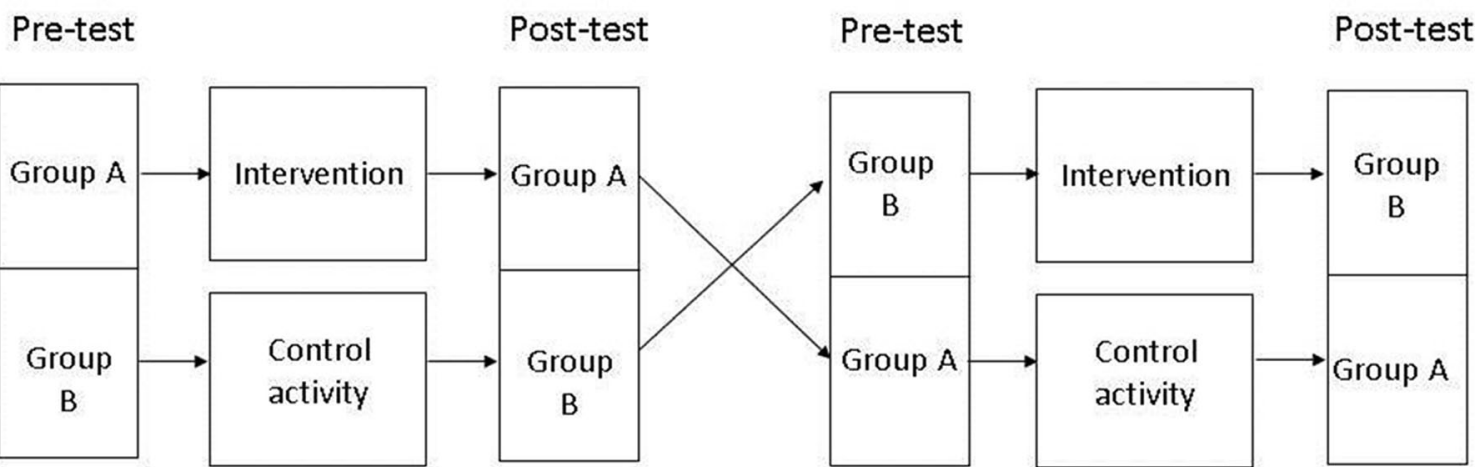

Fig. 1 Cross-over design applied in Study 1 (physical intervention) and Study 2 (mindfulness intervention)

(video presentation), followed by the posttest (15 min, same procedure as that used in the pretest). On the second day, students completed the same assessment with a reversed group assignment (see above and Fig. 1). Assessments followed standardized instruction manuals.

Study 1: Physical Exercise The physical exercise condition was a standardized 15-min physical exercise regimen with a professional trainer, providing instructions and serving as a role model. The intervention included four phases: warm-up, partner-balance exercises, moderate cardiovascular activation induced by interval training, and cool-down. The 2-min warm-up included stretching exercises of arms and upper body. Afterwards, students engaged in a partner exercise, training physical balance $(4 \mathrm{~min})$, then performed $5 \mathrm{~min}$ of interval training including knee-bends, table-dips, and skipping ( 5 blocks of training interspersed with $30 \mathrm{~s}$ of rest), followed by two rounds of running on the spot $(2 \times 20 \mathrm{~s}$ with $20 \mathrm{~s}$ of rest between rounds). For cool-down, participants stretched arms and legs and performed breathing exercises.

Study 2: Mindfulness Training The mindfulness condition was a standardized 15-min intervention instructed by an experienced mindfulness trainer. The first part of the session included easy yoga exercises ( $2 \mathrm{~min}$ ) and the second part included a mindfulness meditation and body scan (13 min). The body scan is a classic mindfulness exercise suitable for novices. For body scan, students were instructed to focus on their breath, bodily sensations, and background noises as well as their own thoughts and mind-wandering. During the mindfulness intervention, participants sat on yoga mats or optionally on chairs. The mindfulness session was instructed by the trainer serving as a role model. Standardized and timed instructions of the body scan were pre-taped and played during the mindfulness intervention.

Control Condition In both studies, participants in the active control conditions watched a video for $15 \mathrm{~min}$ without further instructions. In Study 1 (physical exercise), students in the control condition watched a professional fitness workout with exercises similar to those performed by the intervention condition. In Study 2 (mindfulness training), students watched a yoga documentary.

\section{Measures}

Mood Multidimensional Mood State Questionnaire (Mehrdimensionaler Befindlichkeitsfragebogen, MDBF; Steyer et al. 1994). The MDBF standardized paper-pencil questionnaire assesses three mood dimensions: good-bad, awake-tired, and calm-nervous. Participants are asked to rate their current mood state by 12 items (four for each mood dimension) on a 5-point scale ranging from 1 (not at all) to 5 (very strongly) (e.g., "At the moment I feel good"). Higher values represent better, calmer, or more awake moods. The dependent variables were the raw scores for each subscale.

Attention Frankfurt Attention Inventory-2 (Frankfurter Aufmerksamkeits-Inventar-2, FAIR-2; Moosbrugger et al. 2011). The FAIR-2 is a visual discrimination task and participants are instructed to respond to relevant information while disregarding irrelevant or distracting information. The 6-min timed paper-pencil test consists of 640 highly similar stimuli (four different symbols). Participants are instructed to mark two target stimuli while ignoring other stimuli. The three dependent variables are the performance score (the number of completed items corrected for the number of errors), the quality score (the performance score relative to the total number of completed items), and the continuity score (performance score times quality score). Following the test manual, three participants were excluded from the analyses (two in Study 1 and one in Study 2) because the ratio of errors to the total number of items was larger than 5\%, indicating that they did not follow the instructions properly (cf. Moosbrugger et al. 2011). The dependent variables were the raw scores of the performance, quality, and continuity score. 
Executive Functions The Trail Making Test B (TMT, Reitan 1992) served to assess shifting as one core component of executive functioning. In the paper-pencil test, participants were instructed to draw a line and connect letters and numbers in alternating sequence (i.e., 1-A-2-B-3-C...). Letters and numbers were randomly distributed across the test sheet. In order to minimize retest effects at pretest and posttest, we used parallel versions of the TMT (cf. Toyokura et al. 2003). The TMT was timed to $30 \mathrm{~s}$ and the dependent variable was the total number of correctly completed items.

Demographics and Control Variables Before pretest on day 1, participants provided personal information regarding age, gender, native language, type of teacher training (elementary school, secondary school), and study majors. After the physical intervention (Study 1) and before posttest, participants answered an additional short questionnaire rating their perceived fitness level, the exercise difficulty, and their motivation during the intervention on a 5-point scale ranging from 1 for "not at all" to 5 for "very much." After the mindfulness intervention posttest in Study 2, participants completed a short questionnaire rating their expertise in mindfulness, the exercise difficulty, and their motivation during the intervention on a 5-point scale ranging from 1 for "not at all" to 5 for "very much".

\section{Data Analyses}

Data analyses were performed using SPSS Statistics 27.0. Results were considered significant at $p<.05$. To test for baseline differences at pretest, we ran univariate analyses of variance (ANOVA) with the between-subjects factor group (intervention, control) for age and all dependent variables (i.e., mood, attention, executive function) on days 1 and 2. We also tested for session order effects by running repeatedmeasures ANOVAs with the between-subjects factors group (intervention, control) and session order (intervention first, intervention second) and the within-subjects factor session (pretest, posttest). To test for intervention-related changes, we ran repeated-measures of variances (ANOVAs) with the within-subjects factor session (pretest, posttest) and the between-subjects factor group (intervention, control) for all dependent variables (i.e., mood, attention, executive functions). We report Cohen's $d$ to quantify pretest-posttest differences.

\section{Results}

\section{Controlling for Baseline Differences and Order Effects}

There were no significant baseline differences between conditions for any of the dependent variables (all $p$ values $>.05$ ).
The ANOVAs ran to control for session order effects did not yield significant effects for any of the dependent variables (group $\times$ session $\times$ session order, all $p$ values $>.05$ ). Thus, all further analyses were collapsed across session order.

\section{Study 1: Physical Exercise}

Table 1 presents the means and standard deviations at pretest and posttest for all dependent variables, and effect sizes for pre-post differences (gain) of all dependent variables as well as ANOVA results for the group $\times$ time interaction effects.

\section{Mood}

There was a significant main effect of session for the awake-tired mood dimension, $F(1,62)=9.24, p<.003$, indicating that participants felt more awake at posttest. We also found significant main effects of group for goodbad, $F(1,62)=26.07, p<.001$, and awake-tired, $F(1,62)=$ $49.09, p<.001$, indicating differences between conditions. Importantly, we found significant interactions between session and group for all three mood dimensions (see Table 1). Post hoc comparisons revealed that participants in the intervention condition reported to feel better and more awake at posttest (good-bad: $p<.001$, awake-tired $p<.001$ ), while there were no changes in the control condition (good-bad: $p$ $=.176$, awake-tired $p=.248$ ). None of the conditions showed significant pretest-posttest changes on the mood dimension calm-nervous (intervention condition: $p=.116$, control condition: $p=.058$ ). Effect sizes (Cohen 1992) for the interaction effects were medium to large $\left(\mathrm{eta}^{2}=\right.$ $.08-.30)$.

\section{Attention}

We found significant main effects of session for all attention scores, i.e. increased test scores from pretest to posttest, (performance: $F(1,62)=733.20, p<.001$; quality: $F(1,62)=$ $28.86, p<.001$; continuity: $F(1,62)=622.20, p<.001)$ but no significant main effects of group. Importantly, there were significant interactions between session and group for attention performance and attention continuity (see Table 1). Post hoc comparisons revealed that participants in the intervention and control condition improved significantly between pre- and posttest on attention-performance and attention-continuity of the FAIR test (all $p$-values: <.001) but the improvement in the intervention condition was larger (attention-performance gain: 110.52 versus 76.05 , attention-continuity gain: 111.99 versus 77.06) (see Table 1). Interaction effects were medium-sized $\left(\right.$ eta $\left.^{2}=.11\right)$. 
Table 1 Study 1 (physical exercise): means (SD) for mood, attention, and executive function as a function of condition (intervention, control) and session (pretest, posttest); gain scores (posttest-pretest), ANOVA results, and effect sizes

\begin{tabular}{|c|c|c|c|c|c|c|c|c|c|c|c|}
\hline \multirow[t]{2}{*}{$N$ total $=63$} & \multicolumn{4}{|l|}{ Physical exercise } & \multicolumn{3}{|l|}{ Control condition } & \multicolumn{4}{|c|}{ Group $\times$ time } \\
\hline & Pretest $M(\mathrm{SD})$ & Posttest $M$ (SD) & Gain & $d$ & Pretest $M(\mathrm{SD})$ & Posttest $M(\mathrm{SD})$ & Gain & $d$ & $F$ & $p$ & eta $^{2}$ \\
\hline \multicolumn{12}{|l|}{ Mood } \\
\hline Good-bad & $15.92(2.93)$ & $17.06(2.46)$ & 1.14 & 0.42 & $15.37(3.06)$ & $14.87(2.87)$ & -0.50 & -0.17 & 12.420 & .001 & .17 \\
\hline Awake-tired & $12.27(3.70)$ & $15.02(2.73)$ & 2.75 & 0.85 & $11.30(3.86)$ & $10.79(3.49)$ & -0.51 & -0.14 & 26.479 & .000 & .30 \\
\hline Calm-nervous & $14.56(3.09)$ & $15.22(3.12)$ & 0.66 & 0.21 & $14.59(3.30)$ & $13.94(3.29)$ & -0.65 & -0.20 & 5.642 & .021 & .08 \\
\hline \multicolumn{12}{|l|}{ Attention } \\
\hline Performance & $429.27(119.84)$ & $539.79(90.37)$ & 110.52 & 1.04 & $466.41(118.54)$ & $542.46(87.19)$ & 76.05 & 0.73 & 7.363 & .009 & .11 \\
\hline Quality & $0.95(0.04)$ & $0.96(0.03)$ & 0.01 & 0.28 & $0.95(0.04)$ & $0.96(0.03)$ & 0.01 & 0.28 & $<1$ & .376 & .01 \\
\hline Continuity & $409.82(123.42)$ & $521.81(95.99)$ & 111.99 & 1.01 & $446.24(122.62)$ & $523.30(92.50)$ & 77.06 & 0.71 & 7.458 & .008 & .11 \\
\hline \multicolumn{12}{|l|}{ Shifting } \\
\hline$n$ correct & $13.78(3.86)$ & $16.98(4.51)$ & 3.2 & 0.77 & $13.59(4.09)$ & $16.70(4.94)$ & 3.11 & 0.69 & $<1$ & .900 & .00 \\
\hline
\end{tabular}

Means, SD, and analyses are collapsed across day 1 and day 2 (day 1: physical exercise $n=29$; control condition: $n=34$ ); mood: higher values represent better, calmer, and more awake mood; attention scores: performance $=$ total count of marked signs corrected of errors, quality $=$ performance/total number of completed items, continuity $=$ performance $\times$ quality; gain $=$ performance difference $($ posttest - pretest $) ; d=$ within-group effect size $($ Cohen's $d)$

\section{Executive Functions}

ANOVA results showed a significant main effect of session, i.e., improvements in performance from pretest to posttest, $F(1,62)=59.46, p<.001$, but no further effects reached significance.

\section{Study 2: Mindfulness Intervention}

Table 2 presents the means and standard deviations at pretest and posttest for all dependent variables, effect sizes for pre- post differences (gains) of all dependent variables, and ANOVA results for the group $\times$ time interaction.

\section{Mood}

We only found a significant main effect of session for calmnervous, $F(1,27)=9.44, p<.005$, indicating that participants felt calmer at posttest, but no main effect of group on any other mood dimension. Importantly, there were significant interactions between session and group for all three mood dimensions (see Table 2). Post hoc comparisons revealed that all

Table 2 Study 2 (mindfulness intervention): means (SD) for mood, attention, and executive function as a function of condition (intervention, control) and session (pretest, posttest); gain scores (posttest-pretest), ANOVA results, and effect sizes

\begin{tabular}{|c|c|c|c|c|c|c|c|c|c|c|c|}
\hline \multirow[t]{2}{*}{$N$ total $=28$} & \multicolumn{4}{|c|}{ Mindfulness training } & \multicolumn{4}{|l|}{ Control condition } & \multicolumn{3}{|c|}{ Group $\mathrm{x}$ time } \\
\hline & Pretest & Posttest $M$ (SD) & Gain & $d$ & Pretest $M(\mathrm{SD})$ & Posttest $M(\mathrm{SD})$ & Gain & $d$ & $F$ & $p$ & eta $^{2}$ \\
\hline \multicolumn{12}{|l|}{ Mood } \\
\hline Good-bad & $15.36(3.11)$ & $16.57(2.73)$ & 1.21 & 0.41 & $15.96(2.25)$ & $15.75(2.52)$ & -0.21 & -0.09 & 4.580 & .042 & .15 \\
\hline Awake-tired & $10.68(4.19)$ & $12.54(3.52)$ & 1.86 & 0.48 & $11.43(3.27)$ & $11.46(3.53)$ & 0.03 & 0.01 & 11.292 & .002 & .30 \\
\hline Calm-nervous & $14.00(2.96)$ & $16.32(2.65)$ & 2.32 & 0.83 & $14.75(2.59)$ & $14.61(2.63)$ & -0.14 & -0.05 & 10.459 & .003 & .28 \\
\hline \multicolumn{12}{|l|}{ Attention } \\
\hline Performance & $469.82(127.02)$ & $569.68(88.97)$ & 99.86 & 0.91 & $493.96(115.44)$ & $558.50(87.58)$ & 64.54 & 0.63 & 2.267 & .144 & .08 \\
\hline Quality & $0.95(0.04)$ & $0.97(0.03)$ & 0.02 & 0.57 & $0.95(0.04)$ & $0.96(0.03)$ & 0.01 & 0.28 & $<1$ & .794 & .00 \\
\hline Continuity & 450.19 (132.09) & $552.82(92.58)$ & 102.63 & 0.90 & $471.00(117.96)$ & $539.12(92.55)$ & 68.12 & 0.64 & 2.180 & .151 & .08 \\
\hline \multicolumn{12}{|l|}{ Shifting } \\
\hline$n$ correct & $14.50(4.58)$ & $18.50(4.11)$ & 4.00 & 0.94 & $15.21(5.17)$ & $16.89(4.31)$ & 1.68 & 0.36 & 5.346 & .029 & .17 \\
\hline
\end{tabular}

Means, SD, and analyses are collapsed across day 1and day 2 (day 1: mindfulness condition $n=15$; control condition: $n=13$ ); Mood: higher values represent better, calmer, and more awake mood; Attention scores: performance $=$ total count of marked signs corrected of errors, quality $=$ performance/ total number of completed items, continuity $=$ performance $\times$ quality; gain $=$ performance difference $($ posttest - pretest); $d=$ within-group effect size (Cohen's $d$ ) 
mood dimensions in the intervention condition changed significantly between pre- and posttest (good-bad: $p=.017$, awake-tired: $p=.005$, calm-nervous: $p=.001$ ), with participants reporting to feel better, more awake, and more calm. No such changes were present in the control condition (good-bad: $p=.570$, awake-tired: $p=.949$, calm-nervous: $p=.731$ ). Interaction effects were medium to large $\left(\right.$ eta $\left.^{2}=.15-.30\right)$.

\section{Attention}

We found significant main effects of session, i.e., increased test scores from pretest to posttest for all three attention scores (performance: $F(1,27)=169.99, p<.001$; quality: $F(1,27)=$ 17.66, $p<.001$; continuity: $F(1,27)=149.52, p<.001)$. Neither significant main effects of group nor significant interaction effects were present.

\section{Executive Functions}

There was a significant main effect of session, i.e., improvements in performance from pretest to posttest, $F(1,27)=$ 42.76, $p<.001$, as well as a significant interaction between session and group (see Table 2). Post hoc comparisons showed a significant improvement in the intervention $(p=$ $.000)$ and control condition $(p=.011)$, but the gains were larger in the intervention condition (gain: 4.00 versus 1.68 ). Interaction effects were medium-sized $\left(\right.$ eta $^{2}=.17$ ).

For all other additional between-subjects factors (gender, fitness, difficulty of exercises, motivation during exercises), no significant interaction for all dependent variables was found in Study 1 or Study 2 ( $p>.05$, respectively).

\section{Discussion}

This study tested the effects of brief physical exercise and mindfulness training interventions on university students' mood, attention, and executive function in the classroom. Our results show that a single bout of physical exercise improved mood and attention and a single session of mindfulness improved mood and executive function, indicating that even short physical or mindfulness activities may increase affective and cognitive processes that have been linked to positive learning outcomes (Meltzer 2018; Zins and Goleman 2004).

\section{Effects on Mood}

Individuals in both intervention conditions improved more than those in the control conditions on all mood dimensions. This overall increase in mood is in line with previous results showing improved mood after physical activation (Hansen et al. 2001; Scully et al. 1998; Wichers et al. 2012) and after brief bouts of physical exercise (Hogan et al. 2013; Reed and Ones 2006). Our results are also consistent with previous mindfulness interventions focusing on mood-related outcomes such as stress reduction, emotional stability, and mindfulness itself (Hill and Updegraff 2012; Zeidan et al. 2010). Even though there was a general increase in mood in both studies, the present findings suggest that the interventions may have differential effects on mood. Participants reported being more awake after the physical activity and calmer after the mindfulness activity. However, given that we did not directly compare the results of studies 1 and 2 , this finding needs further confirmation. Considering that mood has been linked to learning outcomes in many contexts (Bryan et al. 1996; Isen 1999), positive effects across different mood dimensions found in the present study indicate that physical and mindfulness training may contribute to improving affective conditions for learning and teaching.

\section{Effects on Attention}

After the physical intervention, attention performance and attention continuity improved more than those in the control condition, while attention quality (accuracy) was not affected. This points to a quantitative (indicated by increased speed) rather than a qualitative (indicated by higher accuracy) improvement of performance, adding to the mixed outcomes of previous studies. Our results support findings from reviews reporting positive effects of short, moderately intense physical exercises (Chang et al. 2012; Lambourne and Tomporowski 2010). There is also previous evidence showing that physical activity improved speed but not accuracy in attention tests (Gallotta et al. 2015; Schmidt et al. 2016). Yet, Budde et al. (2008) found both improved attentional speed and accuracy after physical exercise. However, this study examined elite athletes and findings may not be indicative of our study population of university teacher-students. Moreover, metaanalytic evidence showed that speed of processing and not accuracy seems to be responsible for a large proportion of explained variance of improved attention (McMorris and Hale 2012). Obviously, our results are inconsistent with previous studies which found no evidence for improved attention after 5- or 12-min physical activation in school settings (Kubesch et al. 2009; Van de Weijer-Bergsma et al. 2014) or after 5- or 15-min physical exercise, respectively, in a university setting (Bailey et al. 2014; Hey et al. 2012). Inconsistent results might be explained by differences in design and methodology across studies, such as differences in levels of physical intensity, duration, and intervention content. Van den Berg et al. (2016), for instance, implemented exercises with low-to-moderate intensity, and other studies implementing interventions with at least moderate intensity levels are in line with our results (e.g., Niemann et al. 2013). Hence, moderate exercise might be necessary to yield 
improvements in cognitive performance (for a meta-analysis, see McMorris and Hale 2012). We realize, of course, that we did not measure the intensity of physical activity (e.g., heart rate) in the present study, so this assumption clearly needs to be tested in future research.

In line with other studies, we found no effects of the mindfulness intervention on attention (Jensen et al. 2012). However, the results of our mindfulness intervention are clearly limited because of the small sample size $(N=28)$. Still, our study adds new insights because the evidence for the effects of single-session mindfulness training interventions on attention is scarce. The few existing study results are heterogeneous (e.g., Jensen et al. 2012; Taraban et al. 2017) and have not been implemented in the classroom. We acknowledge further that we focused on a very specific aspect of attention and further research may follow up on our findings and adopt a broader approach by investigating different aspects of attention, such as selective, divided, sustained, vigilant attention and mind-wandering. Our results across studies showed that physical activity but not mindfulness improved selective attention, which may be related to the specificity of the interventions but needs further investigation.

\section{Effects on Executive Functions}

We found no significant effects on executive control following physical exercise. This result is not in line with other studies reporting that even short bouts of physical activation enhanced executive functions (Tomporowski et al. 2015; Verburgh et al. 2014). Given that only a part of the 15-min training involved cardiovascular exercises, the training intensity may not have been high enough to induce effects on higher-order control functions.

After the mindfulness intervention, however, we found positive effects on executive control which is not in line with previous studies involving younger adults (Dunning et al. 2019) but is in line with results found in older adults (Gallant, 2016). We assume that the presently used TMT has a good fit with the mindfulness intervention because it taps several core dimensions of executive control (shifting between letters and numbers, inhibiting the irrelevant dimension, updating the letter-number sequence in working memory). Thus, the mindfulness training might have benefitted performance on this task. During the mindfulness intervention, participants were asked to focus, to constantly inhibit internal and external distraction, and to redirect attention (Allen et al. 2012; Baer 2010; Baer and Krietemeyer 2010; Bishop et al. 2004; Lutz et al. 2008), which can be seen as indicators for a good fit with the tasks on the TMT. The effects of mindfulness interventions on executive functions, however, are certainly complex (Gallant, 2016) and might depend on other factors than those measured in our and other studies (e.g., the group setting of the testing, motivational aspects).

\section{Underlying Processes}

Our study adds to numerous previous studies that have provided evidence for positive effects of physical exercise, particularly cardiovascular training, on a wide range of cognitive abilities (see Colcombe et al. 2004, for a meta-analysis; Pothier and Bherer 2016, for a review). These positive effects of cardiovascular fitness on cognitive abilities have been explained by training-induced changes in the brain. However, most of the studies adding to this literature focused on longterm interventions and not on short bouts of physical activity (e.g., Berkman et al. 2014; Johann et al. 2016). The fact that our results as well as existing studies on single-session physical and mindfulness interventions showed positive effects on both cognition and mood might be explained by overlapping neural representations of specific cognitive and affective processes (Luu \& Hall, 2017). However, further research is needed to disentangle the mechanisms involved and to test specific processes of change (Tang et al. 2015).

\section{Limitations and Future Research Directions}

Limitations of the present studies were that sample sizes of studies 1 and 2 differed, that the sample in Study 2 was relatively small $(N=28)$, and that the gender distribution was unequal (Study 1: $81 \%$ female; Study 2: $68 \%$ female). Our aim was to ensure a high comparability across studies (only teacher-students, same course content) and access to this population was limited. Nevertheless, a post hoc statistical power analysis revealed good statistical power for all dependent variables. Despite the advantages of the cross-over design, participants were only blind regarding the study conditions at the pretest of day 1.

Future studies may consider applying more sensitive measures to assess executive functioning than the trail-making test, which does not allow the differentiation between distinct components of executive control, such as working memory, shifting, and inhibition. Computer-based tests can also provide more finegrained information on reaction times and accuracy. It should also be noted that even though the attention measure (FAIR-2) has shown very good retest reliabilities for such short retest intervals (5 and more minutes), data on the retest reliability for the executive function measure (trail-making test) and the mood questionnaire (MDBF) are also very good, but based on much longer retest intervals (several weeks).

Furthermore, future studies may also adopt a fully randomized allocation to experimental conditions (without matching for gender) and choose another type of control activity that is more comparable to the activities in the intervention conditions. Although watching a video has been used as control activity in similar studies (e.g., Spitzer \& Furtner, 2016) and has methodological advantages as all participants are having similar information input, it may be more appropriate to include control 
conditions that perform some sort of (physical) activity that does not tap the processes targeted in the intervention activities.

In order to investigate effects of classroom-based interventions, more studies in different class contexts are needed. Future classroom-based studies may also include assessments of students' academic performance rather than just testing cognitive performance. Regarding the assessment of physical and mindfulness activities, future studies may choose to include more objective measures, e.g., by using accelerometers (cf. EstebanCornejo et al. 2015). Finally, future studies should also investigate in more detail the dose-response relationship and whether specific physical activities or mindfulness or a combination yield larger benefits for specific cognitive abilities (Chang et al. 2012).

Authors' Contributions CM led the research project, designed and executed the study, analyzed the data, and wrote most of the paper. DD executed the study and contributed to the development of the physical activity intervention. EK assisted in the data analysis and wrote part of the results section. ML and ES executed the study, assisted in the data analyses, and contributed to the writing of the manuscript. NS assisted in the data analysis and wrote part of the Results section. CM contributed to the study design and the development of the interventions. JK co-led the study, contributed to the study design, and edited the final manuscript.

Funding Open Access funding enabled and organized by Projekt DEAL.

\section{Declarations}

Ethical Approval All procedures performed in studies involving human participants were in accordance with the ethical standards of the institutional and/or national research committee and with the 1964 Helsinki Declaration and its later amendments or comparable ethical standards.

Informed Consent Written informed consent was obtained from all participants included in the studies.

Conflict of Interest The authors declare no competing interests.

Open Access This article is licensed under a Creative Commons Attribution 4.0 International License, which permits use, sharing, adaptation, distribution and reproduction in any medium or format, as long as you give appropriate credit to the original author(s) and the source, provide a link to the Creative Commons licence, and indicate if changes were made. The images or other third party material in this article are included in the article's Creative Commons licence, unless indicated otherwise in a credit line to the material. If material is not included in the article's Creative Commons licence and your intended use is not permitted by statutory regulation or exceeds the permitted use, you will need to obtain permission directly from the copyright holder. To view a copy of this licence, visit http://creativecommons.org/licenses/by/4.0/.

\section{References}

Ainley, M. (2006). Connecting with learning: motivation, affect and cognition in interest processes. Educational Psychology Review, 18(4), 391-405. https://doi.org/10.1016/j.brainres.2012.02.068.
Allen, M., Dietz, M., Blair, K. S., van Beek, M., Rees, G., VestergaardPoulsen, P., Lutz, A., \& Roepstorff, A. (2012). Cognitive-affective neural plasticity following active-controlled mindfulness intervention. Journal of Neuroscience, 32(44), 15601-15610. https://doi. org/10.1523/JNeursosci.2957-12.2012.

Allport, D. A. (1980). Attention and performance. In G. Claxton (Ed.), Cognitive Psychology: New Directions (112-153). Routledge \& Kegan.

Ashby, F. G., Isen, A. M., \& Turken, A. U. (1999). A neuropsychological theory of positive affect and its influence on cognition. Psychology Review, 106(3), 529-550. https://doi.org/10.1037/0033-295X.106. 3.529 .

Baer, R. A. (Ed.). (2010). Assessing mindfulness and acceptance processes in clients: Illuminating the theory and practice of change. New Harbinger Publications.

Baer, R. A., \& Krietemeyer, J. (2010). Overview of mindfulness-and acceptance-based treatment approaches. In R. A. Baer (Ed.), Assessing mindfulness and acceptance processes in clients: Illuminating the theory and practice of change (3-27). New Harbinger Publications.

Bailey, E. K., Douglas, T. J., Wolff, D., \& Bailey, S. (2014). Coordinated and aerobic exercise do not improve attention in graduate students. The Open Sports Sciences Journal, 7(1), 203-207.

Barenberg, J., Berse, T., \& Dutke, S. (2011). Executive functions in learning processes: do they benefit from physical activity? Educational Research Review, 6(3), 208-222.

Berkman, E. T., Kahn, L. E., \& Merchant, J. S. (2014). Training-induced changes in inhibitory control network activity. Journal of Neuroscience, 34(1), 149-157.

Berse, T., Rolfes, K., Barenberg, J., Dutke, S., Kuhlenbäumer, G., Völker, K., Winter, B., Wittig, M., \& Knecht, S. (2015). Acute physical exercise improves shifting in adolescents at school: evidence for a dopaminergic contribution. Frontiers in Behavioral Neuroscience, 9, 196. https://doi.org/10.3389/fnbeh.2015.00196.

Bishop, S. R., Lau, M. A., Shapiro, S., Carlson, L., Anderson, N. D., Carmody, J., Segal, Z. V., Abbey, S., Speca, M., \& Velting, D. (2004). Mindfulness: a proposed operational definition. Clinical Psychology: Science and Practice, 11(3), 230-241. https://doi.org/ 10.1093/clipsy.bph077.

Brisswalter, J., Collardeau, M., \& René, A. (2002). Effects of acute physical exercise characteristics on cognitive performance. Sports Medicine, 32(9), 555-566.

Bryan, T., Mathur, S., \& Sullivan, K. (1996). The impact of positive mood on learning. Learning Disability Quarterly, 19(3), 153-162. https://doi.org/10.2307/1511058.

Budde, H., Voelcker-Rehage, C., Pietrabyk-Kendziorra, S., Ribeiro, P., \& Tidow, G. (2008). Acute coordinative exercise improves attentional performance in adolescents. Neuroscience Letters, 441(2), 219-223.

Cahn, B. R., \& Polich, J. (2006). Meditation states and traits: EEG, ERP, and neuroimaging studies. Psychological Bulletin, 132(2), 180-211.

Chang, Y.-K., Labban, J. D., Gapin, J. I., \& Etnier, J. L. (2012). The effects of acute exercise on cognitive performance: a meta-analysis. Brain Research, 1453, 87-101. https://doi.org/10.1016/j.brainres. 2012.02.068

Chen, A.-G., Yan, J., Yin, H.-C., Pan, C.-Y., \& Chang, Y.-K. (2014). Effects of acute aerobic exercise on multiple aspects of executive function in preadolescent children. Psychology of Sport and Exercise, 15(6), 627-636. https://doi.org/10.1016/j.psychsport. 2014.06.004

Chen, S.-R., Tseng, C.-L., Kuo, S.-Y., \& Chang, Y.-K. (2016). Effects of a physical activity intervention on autonomic and executive functions in obese young adolescents: a randomized controlled trial. Health Psychology, 35(10), 1120-1125.

Chong, C. S. M., Tsunaka, M., \& Chan, E. P. (2011). Effects of yoga on stress management in healthy adults: a systematic review. Alternative Therapies in Health and Medicine, 17(1), 32-38. 
Cohen, J. (1992). A power primer. Psychological Bulletin, 112(1), 155159.

Colcombe, S. J., Kramer, A. F., Erickson, K. I., Scalf, P., McAuley, E., Cohen, N. J., Webb, A., Jerome, G. J., Marquez, D. X., \& Elavsky, S. (2004). Cardiovascular fitness, cortical plasticity, and aging. Proceedings of the National Academy of Sciences of the United States of America, 101(9), 3316-3321.

Cramer, H., Lauche, R., Langhorst, J., \& Dobos, G. (2013). Yoga for depression: a systematic review and meta-analysis. Depression and Anxiety, 30(11), 1068-1083.

Driver, J. (2001). A selective review of selective attention research from the past century. British Journal of Psychology, 92(1), 53-78.

Dunning, D. L., Griffiths, K., Kuyken, W., Crane, C., Foulkes, L., Parker, J., \& Dalgleish, T. (2019). Research review: the effects of mindfulness-based interventions on cognition and mental health in children and adolescents - a meta-analysis of randomized controlled trials. Journal of Child Psychology and Psychiatry, 60(3), 244-258. https://doi.org/10.1111/jcpp.12980.

Erisman, S. M., \& Roemer, L. (2010). A preliminary investigation of the effects of experimentally induced mindfulness on emotional responding to film clips. Emotion, 10(1), 72-82. https://doi.org/10. 1037/a0017162.

Esteban-Cornejo, I., Tejero-Gonzalez, C. M., Sallis, J. F., \& Veiga, O. L. (2015). Physical activity and cognition in adolescents: a systematic review. Journal of Science and Medicine in Sport, 18(5), 534-539.

Farley, J., Risko, E. F., \& Kingstone, A. (2013). Everyday attention and lecture retention: the effects of time, fidgeting, and mind wandering. Frontiers in Psychology, 4(619), 1-9. https://doi.org/10.3389/fpsyg. 2013.00619.

Faul, F., Erdfelder, E., Lang, A.-G., \& Buchner, A. (2007). G* Power 3: a flexible statistical power analysis program for the social, behavioral, and biomedical sciences. Behavior Research Methods, 39(2), 175191.

Fennell, A. B., Benau, E. M., \& Atchley, R. A. (2016). A single session of meditation reduces of physiological indices of anger in both experienced and novice meditators. Consciousness and Cognition, 40, 54 66. https://doi.org/10.1016/j.concog.2015.12.010.

Fjorback, L. O., Arendt, M., Ørnbøl, E., Fink, P., \& Walach, H. (2011). Mindfulness-based stress reduction and mindfulness-based cognitive therapy - a systematic review of randomized controlled trials. Acta Psychiatrica Scandinavica, 124(2), 102-119.

Gallant, S. N. (2016). Mindfulness meditation practice and executive functioning: breaking down the benefit. Consciousness and Cognition, 40, 116-130. https://doi.org/10.1016/j.concog.2015.12. 010

Gallotta, M. C., Emerenziani, G. P., Franciosi, E., Meucci, M., Guidetti, L., \& Baldari, C. (2015). Acute physical activity and delayed attention in primary school students. Scandinavian Journal of Medicine \& Science in Sports, 25(3), e331-e338.

Goldberg, S. B., Tucker, R. P., Greene, P. A., Simpson, T. L., Kearney, D. J., \& Davidson, R. J. (2017). Is mindfulness research methodology improving over time? A systematic review. PLoS One, 12(10: e0187298), 1-16. https://doi.org/10.1371/journal.pone.0187298.

Gothe, N., Pontifex, M. B., Hillman, C., \& McAuley, E. (2013). The acute effects of yoga on executive function. Journal of Physical Activity \& Health, 10(4), 488-495.

Hansen, C. J., Stevens, L. C., \& Coast, J. R. (2001). Exercise duration and mood state: how much is enough to feel better? Health Psychology, 20(4), 267-275.

Hey, S., Löffler, S. N., Walter, K., Grund, A., König, N. E. W., \& Bös, K. (2012). Kurzzeitige aktive und passive Regenerationspausen. Prävention Und Gesundheitsförderung, 7(2), 120-126. https://doi. org/10.1007/s11553-012-0332-7.

Hill, C. L. M., \& Updegraff, J. A. (2012). Mindfulness and its relationship to emotional regulation. Emotion, 12(1), 81.
Hofmann, S. G., Sawyer, A. T., Witt, A. A., \& Oh, D. (2010). The effect of mindfulness-based therapy on anxiety and depression: a metaanalytic review. Journal of Consulting and Clinical Psychology, 78(2), 169-183.

Hogan, C. L., Mata, J., \& Carstensen, L. L. (2013). Exercise holds immediate benefits for affect and cognition in younger and older adults. Psychology and Aging, 28(2), 587-594. https://doi.org/10. 1037/a0032634.

Isen, A. M. (1999). On the relationship between affect and creative problem solving. In S. W. Russ (Ed.), Affect, Creative Experience and Psychological Adjustment (Vol. 3, pp. 3-17). Taylor \& Francis.

Jäger, K., Schmidt, M., Conzelmann, A., \& Roebers, C. M. (2015). The effects of qualitatively different acute physical activity interventions in real-world settings on executive functions in preadolescent children. Mental Health and Physical Activity, 9, 1-9. https://doi.org/10. 1016/j.mhpa.2015.05.002.

Janssen, M., Chinapaw, M. J. M., Rauh, S. P., Toussaint, H. M., van Mechelen, W., \& Verhagen, E. (2014). A short physical activity break from cognitive tasks increases selective attention in primary school children aged 10-11. Mental Health and Physical Activity, 7(3), 129-134. https://doi.org/10.1016/j.mhpa.2014.07.001.

Jensen, C. G., Vangkilde, S., Frokjaer, V., \& Hasselbalch, S. G. (2012). Mindfulness training affects attention - or is it attentional effort? Journal of Experimental Psychology: General, 141(1), 106-123. https://doi.org/10.1037/a0024931.

Jha, A. P., Krompinger, J., \& Baime, M. J. (2007). Mindfulness training modifies subsystems of attention. Cognitive, Affective, \& Behavioral Neuroscience, 7(2), 109-119.

Johann, V. E., Stenger, K., Kersten, S., \& Karbach, J. (2016). Effects of motor-cognitive coordination training and cardiovascular training on motor coordination and cognitive functions. Psychology of Sport and Exercise, 24, 118-127. https://doi.org/10.1016/j. psychsport.2016.01.008.

Kabat-Zinn, J. (2003). Mindfulness-based interventions in context: past, present, and future. Clinical Psychology: Science and Practice, 10(2), 144-156. https://doi.org/10.1093/clipsy.bpg016.

Kahneman, D. (1973). Attention and effort. Prentice-Hall.

Kubesch, S., Walk, L., Spitzer, M., Kammer, T., Lainburg, A., Heim, R., \& Hille, K. (2009). A 30-minute physical education program improves students' executive attention. Mind, Brain, and Education, 3(4), 235-242. https://doi.org/10.1111/j.1751-228X.2009.01076.x.

Lambourne, K., \& Tomporowski, P. D. (2010). The effect of exerciseinduced arousal on cognitive task performance: a meta-regression analysis. Brain Research, 1341, 12-24.

Lavie, N. (2005). Distracted and confused? Selective attention under load. Trends in Cognitive Sciences, 9(2), 75-82.

Lin, K.-Y., Hu, Y.-T., Chang, K.-J., Lin, H.-F., \& Tsauo, J.-Y. (2011). Effects of yoga on psychological health, quality of life, and physical health of patients with cancer: a meta-analysis. Evidence-based Complementary and Alternative Medicine, 659876. https://doi.org/ $10.1155 / 2011 / 659876$.

Lindquist, S. I., \& McLean, J. P. (2011). Daydreaming and its correlates in an educational environment. Learning and Individual Differences, 21(2), 158-167.

Ludyga, S., Gerber, M., Brand, S., Holsboer-Trachsler, E., \& Pühse, U. (2016). Acute effects of moderate aerobic exercise on specific aspects of executive function in different age and fitness groups: a meta-analysis. Psychophysiology, 53(11), 1611-1626. https://doi. org/10.1111/psyp.12736.

Lutz, A., Slagter, H. A., Dunne, J. D., \& Davidson, R. J. (2008). Attention regulation and monitoring in meditation. Trends in Cognitive Sciences, 12(4), 163-169.

Luu, K., \& Hall, P. A. (2017). Examining the acute effects of hatha yoga and mindfulness meditation on executive function and mood. Mindfulness, 8(4), 873-880. 
McMorris, T., \& Hale, B. J. (2012). Differential effects of differing intensities of acute exercise on speed and accuracy of cognition: a meta-analytical investigation. Brain and Cognition, 80(3), 338-351.

Meltzer, L. (2018). Executive function in education: from theory to practice. Guilford Publications.

Miyake, A., Friedman, N. P., Emerson, M. J., Witzki, A. H., Howerter, A., \& Wager, T. D. (2000). The unity and diversity of executive functions and their contributions to complex "frontal lobe" tasks: A latent variable analysis. Cognitive Psychology, 41(1), 49-100.

Mohan, A., Sharma, R., \& Bijlani, R. L. (2011). Effect of meditation on stress-induced changes in cognitive functions. The Journal of Alternative and Complementary Medicine, 17(3), 207-212. https:// doi.org/10.1089/acm.2010.0142.

Moore, A., \& Malinowski, P. (2009). Meditation, mindfulness and cognitive flexibility. Consciousness and Cognition, 18(1), 176-186.

Moosbrugger, H., Oehlschlägel, J., \& Steinwascher, M. (2011). FAIR-2: Frankfurter Aufmerksamkeits-Inventar 2. Hogrefe.

Mrazek, M. D., Smallwood, J., \& Schooler, J. W. (2012). Mindfulness and mind-wandering: finding convergence through opposing constructs. Emotion, 12(3), 442-448.

Nadler, R. T., Rabi, R., \& Minda, J. P. (2010). Better mood and better performance: learning rule-described categories is enhanced by positive mood. Psychological Science, 21(12), 1770-1776. https://doi. org/10.1177/0956797610387441.

Napoli, M., Krech, P. R., \& Holley, L. C. (2005). Mindfulness training for elementary school students: the attention academy. Journal of Applied School Psychology, 21(1), 99-125.

Niemann, C., Wegner, M., Voelcker-Rehage, C., Holzweg, M., Arafat, A. M., \& Budde, H. (2013). Influence of acute and chronic physical activity on cognitive performance and saliva testosterone in preadolescent school children. Mental Health and Physical Activity, 6(3), 197-204.

Nyklíček, I., \& Kuijpers, K. F. (2008). Effects of mindfulness-based stress reduction intervention on psychological well-being and quality of life: is increased mindfulness indeed the mechanism? Annals of Behavioral Medicine: A Publication of the Society of Behavioral Medicine, 35(3), 331-340. https://doi.org/10.1007/s12160-0089030-2.

Pekrun, R., Goetz, T., Daniels, L. M., Stupnisky, R. H., \& Perry, R. P. (2010). Boredom in achievement settings: exploring control-value antecedents and performance outcomes of a neglected emotion. Journal of Educational Psychology, 102(3), 531-539. https://doi. org/10.1037/a0019243.

Penedo, F. J., \& Dahn, J. R. (2005). Exercise and well-being: a review of mental and physical health benefits associated with physical activity. Current Opinion in Psychiatry, 18(2), 189-193.

Pontifex, M. B., Hillman, C. H., Fernhall, B. O., Thompson, K. M., \& Valentini, T. A. (2009). The effect of acute aerobic and resistance exercise on working memory. Medicine \& Science in Sports \& Exercise, 41(4), 927-934.

Pothier, K., \& Bherer, L. (2016). Physical training. In T. Strobach \& J. Karbach (Eds.), Cognitive Training (pp. 145-153). Springer.

Ramstetter, C. L., Murray, R., \& Garner, A. S. (2010). The crucial role of recess in schools. Journal of School Health, 80(11), 517-526. https://doi.org/10.1111/j.1746-1561.2010.00537.x.

Rausch, S. M., Gramling, S. E., \& Auerbach, S. M. (2006). Effects of a single session of large-group meditation and progressive muscle relaxation training on stress reduction, reactivity, and recovery. International Journal of Stress Management, 13(3), 273-290. https://doi.org/10.1037/1072-5245.13.3.273.

Reed, J., \& Ones, D. S. (2006). The effect of acute aerobic exercise on positive activated affect: a meta-analysis. Psychology of Sport and Exercise, 7(5), 477-514.

Reeve, J. (2014). Understanding motivation and emotion. Wiley.

Reitan, R. M. (1992). Trail Making Test: manual for administration and scoring. Reitan Neuropsychology Laboratory.
Roig, M., Nordbrandt, S., Geertsen, S. S., \& Nielsen, J. B. (2013). The effects of cardiovascular exercise on human memory: a review with meta-analysis. Neuroscience \& Biobehavioral Reviews, 37(8), $1645-1666$.

Sauer, S., Lemke, J., Wittmann, M., Kohls, N., Mochty, U., \& Walach, H. (2012). How long is now for mindfulness meditators? Personality and Individual Differences, 52(6), 750-754.

Schmidt, M., Benzing, V., \& Kamer, M. (2016). Classroom-based physical activity breaks and children's attention: cognitive engagement works! Frontiers in Psychology, 7(1474), 1-13. https://doi.org/10. 3389/fpsyg.2016.01474.

Scully, D., Kremer, J., Meade, M. M., Graham, R., \& Dudgeon, K. (1998). Physical exercise and psychological well being: a critical review. British Journal of Sports Medicine, 32(2), 111-120.

Sharp, J. G., Hemmings, B., Kay, R., Murphy, B., \& Elliott, S. (2017). Academic boredom among students in higher education: a mixedmethods exploration of characteristics, contributors and consequences. Journal of Further and Higher Education, 41(5), 657-677.

Slagter, H. A., Lutz, A., Greischar, L. L., Francis, A. D., Nieuwenhuis, S., Davis, J. M., \& Davidson, R. J. (2007). Mental training affects distribution of limited brain resources. PLoS Biology, 5(6).

Spitzer, M. W. H., \& Furtner, M. (2016). Being physically active versus watching physical activity - effects on inhibitory control. Trends in Neuroscience and Education, 5(1), 30-33. https://doi.org/10.1016/j. tine.2016.02.003.

Steyer, R., Schwenkmezger, P., Notz, P., \& Eid, M. (1994). Testtheoretische Analysen des Mehrdimensionalen Befindlichkeitsfragebogen (MDBF). Diagnostica, 40(4), 320-328.

Tang, Y.-Y., Hölzel, B. K., \& Posner, M. I. (2015). The neuroscience of mindfulness meditation. Nature Reviews Neuroscience, 16(4), 213 225.

Taraban, O., Heide, F., Woollacott, M., \& Chan, D. (2017). The effects of a mindful listening task on mind-wandering. Mindfulness, 8(2), 433-443. https://doi.org/10.1007/s12671-016-0615-8.

Titz, C., \& Karbach, J. (2014). Working memory and executive functions: effects of training on academic achievement. Psychological Research, 78(6), 852-868.

Tomporowski, P. D. (2003). Effects of acute bouts of exercise on cognition. Acta Psychologica, 112(3), 297-324. https://doi.org/10.1016/ S0001-6918(02)00134-8.

Tomporowski, P. D., McCullick, B., Pendleton, D. M., \& Pesce, C. (2015). Exercise and children's cognition: the role of exercise characteristics and a place for metacognition. Journal of Sport and Health Science, 4(1), 47-55. https://doi.org/10.1016/j.jshs.2014. 09.003

Toyokura, M., Ishida, A., Watanabe, F., Okada, N., \& Yamazaki, M. (2003). Intermanual difference in the Japanese Trail Making Test and its mirror version: intra-subject comparison of the taskcompletion time, cognitive time, and motor time. Disability and Rehabilitation, 25(24), 1339-1343.

Tyng, C. M., Amin, H. U., Saad, M. N. M., \& Malik, A. S. (2017). The influences of emotion on learning and memory. Frontiers in Psychology, 8(1454), 1-22. https://doi.org/10.3389/fpsyg.2017. 01454.

van Dam, N. T., van Vugt, M. K., Vago, D. R., Schmalzl, L., Saron, C. D., Olendzki, A., Meissner, T., Lazar, S. W., Kerr, C. E., \& Gorchov, J. (2018). Mind the hype: a critical evaluation and prescriptive agenda for research on mindfulness and meditation. Perspectives on Psychological Science, 13(1), 36-61. https://doi. org/10.1177/1745691617709589.

Van de Weijer-Bergsma, E., Langenberg, G., Brandsma, R., Oort, F. J., \& Bögels, S. M. (2014). The effectiveness of a school-based mindfulness training as a program to prevent stress in elementary school children. Mindfulness, 5, 238-248. https://doi.org/10.1007/s12671012-0171-9. 
Van den Berg, V., Saliasi, E., de Groot, R. H. M., Jolles, J., Chinapaw, M. J. M., \& Singh, A. S. (2016). Physical activity in the school setting: cognitive performance is not affected by three different types of acute exercise. Frontiers in Psychology, 7(723), 1-9. https://doi. org/10.3389/fpsyg.2016.00723.

Verburgh, L., Königs, M., Scherder, E. J. A., \& Oosterlaan, J. (2014). Physical exercise and executive functions in preadolescent children, adolescents and young adults: a meta-analysis. British Journal of Sports Medicine, 48(12), 973-979. https://doi.org/10.1136/ bjsports-2012-091441.

Verhaeghen, P. (2016). Mindfulness and meditation training. In T. Strobach \& J. Karbach (Eds.), Cognitive Training (pp. 127-136). Springer.

Watier, N., \& Dubois, M. (2016). The effects of a brief mindfulness exercise on executive attention and recognition memory. Mindfulness, 7(3), 745-753.

Wichers, M., Peeters, F., Rutten, B. P. F., Jacobs, N., Derom, C., Thiery, E., Delespaul, P., \& van Os, J. (2012). A time-lagged momentary assessment study on daily life physical activity and affect. Health Psychology, 31(2), 135-144. https://doi.org/10.1037/a0025688.

Wilson, K., \& Korn, J. H. (2007). Attention during lectures: beyond ten minutes. Teaching of Psychology, 34(2), 85-89.

Zeidan, F., Johnson, S. K., Diamond, B. J., David, Z., \& Goolkasian, P. (2010). Mindfulness meditation improves cognition: evidence of brief mental training. Consciousness and Cognition, 19(2), 597605.

Zinke, K., Einert, M., Pfennig, L., \& Kliegel, M. (2012). Plasticity of executive control through task switching training in adolescents. Frontiers in Human Neuroscience, 6, 41. https://doi.org/10.3389/ fnhum.2012.00041.

Zins, J. E., \& Goleman, D. (2004). Building academic success on social and emotional learning: what does the research say? The Series on social emotional learning. Teachers College Press.

Publisher's Note Springer Nature remains neutral with regard to jurisdictional claims in published maps and institutional affiliations. 